(2) $\begin{array}{r}\begin{array}{r}\text { BETA } \\ \text { Bureau } \\ \text { d'économie } \\ \text { théorique }\end{array} \\ \text { et appliquée }\end{array}$

\title{
« Environmental Quality and Monopoly Pricing »
}

\author{
$\underline{\text { Auteurs }}$ \\ Rabah Amir, Isabelle Maret \\ Document de Travail $n^{\circ} 2018-31$
}

Juillet 2018

Bureau d'Économie

Théorique et Appliquée

BETA

http://www.beta-umr7522.fr

https://twitter.com/beta econo $\underline{\text { mics }}$

Contact : jaoulgrammare@betacnrs.unistra.fr 


\title{
Environmental Quality and Monopoly Pricing*
}

\author{
Rabah Amir ${ }^{\dagger}$ and Isabelle Maret ${ }^{\ddagger}$
}

July 11, 2018

\begin{abstract}
This paper investigates various aspects of a monopolist's pricing and environmental quality choice, as two simultaneous decisions and with each as a separate decision, the other variable being exogenously fixed. Green quality is modeled as in Spence (1975), and the present analysis builds on his pioneering work. We contrast the private and the first-best socially optimal solutions. While the latter follows the intuitive property of assigning a higher price to higher quality, the former solution does so under a natural condition of log-supermodular demand. This condition is studied in some detail, and related to properties of an underlying utilty function. We complete this characterization of optimal pricing by providing two different counter-intuitive examples where the two-dimensional interaction is such that the monopolist ends up charging a lower optimal price than the social planner, as well as producing a lower quality. Finally, we investigate respective sufficient conditions under which (i) the private and first-best solutions coincide, and (ii) the two-dimensional problem reduces to a one-dimensional problem where the firm picks a single quality-price ratio.
\end{abstract}

JEL codes: Q50, L00, D60.

Key words and phrases: environmental quality, green goods, green awareness, multi-distortion monopoly pricing.

${ }^{*}$ This research was partially financed by the "Cercle Gutenberg" via the "Chaire Gutenberg : On environmental R\&D and Imperfect Competition" at the University of Strasbourg, CNRS, BETA UMR 7522, F - 67000 Strasbourg, France.

${ }^{\dagger}$ Department of Economics, University of Iowa (e-mail: rabah-amir@uiowa.edu).

${ }^{\ddagger}$ Université de Strasbourg, Université de Lorraine, CNRS, BETA UMR 7522, F-67000, Strasbourg, France (e-mail: maret@unistra.fr). 


\section{Introduction}

Environmental quality has emerged in recent decades as a crucially important dimension of product differentiation for many consumer goods, ranging all the way from agricultural produce to high-tech products. There is a substantial body of evidence pointing quite unambiguously to the conclusion that a significant proportion of consumers in the industrialized world have a higher willingness to pay for green products, as opposed to traditional or brown products. ${ }^{1}$ It follows that, at equal prices, (virtually) all consumers will prefer a green product to a brown product, or a greener product to a less green product. In other words, environmental quality naturally fits as part of vertical product differentiation in the usual dichotomy used in industrial organization to classify the two main types of product differentiation.

In view of the increasing attention devoted to environmental issues in general, it then comes as no surprise that a number of recent studies in environmental economics have investigated some aspect of environmental quality and its effects on market performance, along with various public policy initiatives. The issues that were considered include among others optimal standards, tax and other types of policy in vertically differentiated duopoly (e.g., Moraga-Gonzalez and Padron-Fumero, 2002, Lombardini-Riipinen, 2005, and Mantovani and Vergari, 2015), the scope of validity of the Porter hypothesis in strategic settings (e.g., André, González and Porteiro, 2009, and Lambertini and Tampieri, 2012a), and the effects of minimal environmental quality standards (e.g., Lambertini and Tampieri, 2012b).

All the aforementioned studies share a common modeling feature: They adopt the now-standard vertical differentiation model pioneered by Mussa and Rosen (1978), and further developed by Gabszewicz and Thisse (1979), Shaked and Sutton (1982). Thus these studies posit some aspects of environmental quality as the (aggregated) vertical attribute. In contrast, the present paper is an attempt to contribute to this strand of literature by adopting Spence's (1975) earlier and pioneering approach of modeling general product quality as a simple way to operationalize product quality. ${ }^{2}$

An exception to the above literature strand is older work by Ebert and von dem Hagen (1998), which models the environmental quality of a polluting product by inserting emissions (as a negative

\footnotetext{
${ }^{1}$ According to a study by OECD (2002), $27 \%$ of consumers in OECD countries may be seen as "green consumers". In addition, a large proportion of consumers refrain from purchasing products sold by firms that are known to be major polluters or that misrepresent the environmetal content of their products.

${ }^{2}$ Although Spence's pioneering approach used to be quite familiar to economists working in industrial organization, as confirmed by the fact that it is covered in Varian's (1992) classical graduate textbook, it is fair to say that it has been eclipsed by the Mussa-Rosen model in recent decades.
} 
measure of quality) as a separate argument in the inverse demand function. These authors then derive the socially optimal Pigouvian tax-subsidy policy for a monopoly market. ${ }^{3}$ In effect, although apparently unaware of Spence's work, these authors have chosen to model environmental quality in a similar manner.

Naturally, the two approaches to modeling product quality are not unambiguously ranked in terms of overall merit; rather, they each have their advantages and shortcomings. When it comes to environmental quality, Spence's model can be seen as advantageous in the following respects: (i) It allows for more varieties (or quality levels) than just two in the tractable version of the MussaRosen model, (ii) It more readily allows for a general approach as opposed to relying on specific functional forms for the model primitives, and (iii) It dispenses with the simplifying (but crucial) assumptions of a covered market and of a unit purchase per consumer. Spence's model is particularly appropriate when products are differentiated in terms of environmental quality, but are otherwise essentially homogeneous. A simple concrete example is bio-produce instead of regular produce, say tomatoes for definiteness. A bio tomato is of higher environmental quality when the hosting soil contains fewer chemicals, the planted seeds include fewer GM ingredients, the fertilizers and the protection agents used have less chemical content, etc...Another class of products captured by Spence's formulation might be automobiles that are similar in terms of both vertical and horizontal traditional attributes (such as horse power, comfort, size, brand name, appearance, color, etc...), but differentiated in terms of environmental performance, as measured e.g., by some normalized measure of emissions. This differentiation could be due to the overall quality/performance of the catalytic converter, and/or to whether it is fuel, hybrid or electric powered.

The main purpose of this paper is to investigate various aspects of a monopoly firm's pricing and environmental quality choice, both when viewed as two simultaneous decisions and when chosen separately one at a time, the other variable being fixed and thus taken as a given parameter. In so doing, we build on the results of Spence (1975) and Sheshinski (1976), after providing a summary of their main results. ${ }^{4}$ These two authors both reasoned in terms of output and quality levels and showed that the two monopoly choices are generally not socially optimal. In this setting, there are two distortions to monopoly pricing, due to the fact that the firm prices with the marginal consumer

\footnotetext{
${ }^{3}$ For a similar excercise dealing with the green services industry under imperfect competition, see David and Sinclair-Desgagné (2010).

${ }^{4}$ There is a noteworthy literature prior to these two classical studies, surveyed by Schmalensee (1979), but it dealt with other issues, such as scale effects in the cost function. We will not really touch upon such issues, in light of the fact that we are adopting the linear cost assumption of Spence (1975).
} 
in mind, while a social planner will consider the average consumer. As a result, at fixed output, the monopolist may under or over provide quality relative to a social planner, depending on whether the marginal valuation of quality is below or above the average valuation of quality. More precisely, under (over)-provision prevails if the cross partial derivative of the inverse demand with respect to output and quality is (negative) positive. These authors then proceed to investigate a number of different regulation schemes that may alleviate the two distortions, as summarized later on in the present paper.

For a preview of our results, we first note that the present treatment of Spence's problem is in terms of price (instead of output) and quality choices. Although both Spence (1975) and Sheshinski (1976) were clearly aware that, when both choices are made simultaneously, the optimal monopoly output may well end up being higher than the social planner's, no actual example of such a scenario was provided in their studies. We complement their analysis by providing two different examples where the two-dimensional interaction is such that the monopolist ends up charging a lower optimal price than the social planner, as well as producing a lower quality. If one thinks of quality choice by a monopoly firm as entailing a second market distortion, then the two distortions are thereby seen not to be necessarily mutually reinforcing. Thus, even such a foregone conclusion in price theory as monopoly over-pricing (relative to a social planner) may be reversed in case there is a concomitant quality choice.

As part of further investigation of the pricing behavior of a monopolist in the setting at hand, we find that, with price held fixed (e.g., as a consequence of a strong form of regulation), the monopolist always under-provides quality relative to the first-best solution. In addition, while a social planner always responds by increasing quality, the firm will do so under a sufficient condition on demand, that it be log-supermodular in price and quality. To establish that the firm may actually respond with a lower quality without this sufficient condition, we provide an example with closedform solutions where the latter, very counter-intuitive, property holds in a global sense. Likewise, if quality is held fixed at some exogenous level (e.g, through regulation in the form of a minimal quality standard) and this level is increased, the firm responds with a higher price under the same condition of log-supermodular demand.

The condition of log-supermodular demand in price and a relevant parameter (a demand shifter) has emerged in other economic contexts and is clearly an important property for a family of demand functions to possess. In particular, it has the exact economic interpretation that the price elasticity of demand is increasing in the parameter, here product quality. In other words, demand 
becomes more inelastic as product quality is increased. This is arguably the most natural definition of what product quality ought to be thought of, whether it is of an environmental sort or otherwise. ${ }^{5}$ Consequently, we provide a micro-economic foundation via the commonly used construct of a representative consumer and derive a condition of the utility function ensuring that the resulting demand function satisfies this natural property.

Another issue analyzed in the present paper is the derivation of sufficient conditions under which the monopolist will provide a first-best socially efficient level of quality (without any regulation). In so doing, we build on Spence's analysis of a class of multiplicative demands where the dependence on price is hyperbolic (elasticity is independent of price and increasing in quality).

The final question we tackle is whether the firm's and the planner's two-dimensional choice problems can both be reduced to one-dimensional problems, where the single (aggregated) decision can then be regarded as a quality-adjusted price. It turns out that from the same afore-mentioned class of demand functions, we find one subclass where this reduction property holds without any loss of optimality for both the firm and the social planner. As similar one-dimensional problems were already considered in the classical vertical differentiation literature (Sutton, 1991, 1998), this provides an interesting link between Spence's formulation and the dominant model.

We conclude this Introduction with two final remarks. The first is that our decision to couch product quality in terms of environmental attributes is motivated primarily by the desire to introduce the strand of literature on product quality pioneered by Spence (1975) to environmental economics. Our conclusions otherwise apply a priori equally well to traditional notions of product quality, and thus should be of interest for the literature on vertical product differentiation in industrial organization as well. The second remark is that we use in a very simple form the results from supermodular optimization, a natural technique here so as to avoid specifying a two-dimensional second-order condition for the optimization problems at hand (see e.g., Vives, 1999).

This paper is organized as follows. Section 2 describes the model. Section 3 is devoted to the analysis of one decision variable at a time, and Section 5 deals with the case of simultaneous choices. Section 4 contains a micro foundation for demand. Section 6 deals with the case where the model reduces to a one-dimensional problem. Section 7 discusses the effects of suitable public policy. Finally, Section 8 contains the proofs.

\footnotetext{
${ }^{5}$ The condition of log-supermodular demand is slightly stronger than the rotation condition introduced by Myatt and Johnson (2006) in their study of the foundations of product design.
} 


\section{The model}

As in the classical Spence (1975) model, consider a monopoly firm with constant unit cost of production operating in a market with a demand function $D(p, a)$, where $p \geq 0$ is the price charged by the firm, and $a$ is a measure of product quality. To produce a product of quality $a$ entails a unit cost of $c(a)$ depending on the chosen quality of its product, $a$.

While Spence conceived of product quality in rather general and abstract terms, in the present paper, we shall think of quality as being of an environmental nature. Thus higher quality will mean that a good was produced with a more environmentally friendly production process. This greener good may be achieved through a new technology or via the use of end-of-pipe filters as part of the old technology. To fix ideas, one may think simply about fruits or vegetables with a bio label of variable degree. The lowest quality would then be the regular (non-green) version of the product, and the quality would then increase as more natural ingredients (and thus fewer artificial inputs) are used in the agricultural process. ${ }^{6}$

In what follows, we shall refer to environmental quality and quality of a general nature (as in Spence, 1975) interchangeably. This is to stress that our analysis and results apply equally well to both settings.

The profit function is then

$$
\pi(p, a)=[p-c(a)] D(p, a) .
$$

The firm chooses simultaneously the quality of its product, $a$, in some given quality space $\left[0, a_{\max }\right]$ and the price, $p \geq 0$, so as to

$$
\max \{\pi(p, a): p \geq 0, a \in[0, A]\}
$$

The following assumptions will be maintained throughout this paper.

(A1) The demand function $D$ is twice continuously differentiable and satisfies $D_{p}(p, a)<0$ and $D_{a}(p, a)>0$ for all $(p, a)$.

(A2) The unit cost function $c:\left[0, a_{\max }\right] \longrightarrow \mathbb{R}_{+}$is twice continuously differentiable and satisfies $c^{\prime}(\cdot) \geq 0$.

These assumptions are standard in the related literature. The part $D_{p}(p, a)<0$ captures the usual law of demand, and $D_{a}(p, a)>0$ means that a higher quality leads to a higher demand curve.

\footnotetext{
${ }^{6}$ For instance, these natural ingredients may include natural grain and fertilizers, which contain no chemicals and no artificial preservatives.
} 
The cost structure reflects constant returns to scale, i.e., $c(a)$ is the constant unit cost. As higher quality is more costly to produce, it is natural to require that $c^{\prime}(\cdot) \geq 0$. Thus, the process of infusing products with greener content is subject to decreasing returns to scale, with the limit case of constant returns to scale still allowed.

Finally, $a_{\max }$ is an exogenously given maximal feasible green quality, given the present state of know-how. This assumption is mostly for technical convenience. Likewise, quality 0 can be thought of as the brown product, corresponding to the status quo when only pure cost minimization, and no environmental considerations, guides the production process.

The firm's first order conditions with respect to $p$ and $a$ are respectively

$$
\left\{\begin{array}{c}
{\left[p^{m}-c\left(a^{m}\right)\right] D_{p}\left(p^{m}, a^{m}\right)+D\left(p^{m}, a^{m}\right)=0} \\
{\left[p^{m}-c\left(a^{m}\right)\right] D_{a}\left(p^{m}, a^{m}\right)-c^{\prime}\left(a^{m}\right) D\left(p^{m}, a^{m}\right)=0 .}
\end{array}\right.
$$

We shall compare the optimal choice(s) of the monopoly firm to the social optimum under several scenarios. Consumer surplus is defined as usual by

$$
S(p, a)=\int_{p}^{+\infty} D(t, a) d t
$$

and total surplus (or social welfare) is defined as usual by

$$
W(p, a)=\int_{p}^{+\infty} D(t, a) d t+[p-c(a)] D(p, a) .
$$

The social planner chooses simultaneously the quality of the product, $a^{s}$, and the price, $p^{s}$, so as to

$$
\max \left\{W(p, a): p \geq 0, a \in\left[0, a_{\max }\right]\right\}
$$

The planner's first order conditions with respect to $p$ and $a$ are respectively

$$
\left\{\begin{array}{c}
{\left[p^{s}-c\left(a^{s}\right)\right] D_{p}\left(p^{s}, a^{s}\right)=0} \\
\int_{p^{s}}^{+\infty} D_{a}\left(t, a^{s}\right) d t+\left[p^{s}-c\left(a^{s}\right)\right] D_{a}\left(p^{s}, a^{s}\right)-c^{\prime}\left(a^{s}\right) D\left(p^{s}, a^{s}\right)=0 .
\end{array}\right.
$$

These conditions can be rewritten as

$$
\left\{\begin{array}{c}
p^{s}=c\left(a^{s}\right) \\
\int_{p^{s}}^{+\infty} D_{a}\left(t, a^{s}\right) d t-c^{\prime}\left(a^{s}\right) D\left(c\left(a^{s}\right), a^{s}\right)=0
\end{array} .\right.
$$

As is well known, the social planner always sets price equal to marginal cost. Since price is also equal to average cost in this setting, the firm achieves no profit under this solution. 


\section{Setting price with exogenous quality and vice-versa}

In this section, we consider the two subproblems where the monopolist chooses one of its two decision variables only, the other one being held fixed at some exogenous level (for reasons to be specified below) throughout this section.

\subsection{The case of fixed quality}

We begin by treating the more important case where the quality level is exogenously fixed, and consider the standard problem of monopoly pricing, namely

$$
\max \{[p-c(a)] D(p, a): p \geq 0\}
$$

We thus investigate the effects of exogenous quality on pricing. This is an interesting question on its own right for consumer and environmental economics. Indeed, since it is generally believed that high quality or green products sell for higher prices, it is worth uncovering exact conditions when this actually happens. At the same time, often, quality may indeed be considered fixed by the firm, due to some regulatory requirements, such as a binding minimal quality standard. Alternatively, product quality may be considered fixed by the firm as a short-run constraint, while product innovation proceeds to determine future versions (or qualities) of the product for the medium or the long-run.

The main results that we shall prove in this section are as follows.

(i) For any given quality level the monopoly price is necessarily larger than the socially optimal price.

(ii) While price and quality are always complements at the social level, they are complements at the private level for a broad class of demand functions, but not a universal one.

Denote by $p^{m}(a)$ the optimal price response correspondence of the monopolist as quality changes exogenously. ${ }^{7}$ Denote the corresponding map for the social planner by $p^{s}(a)$.

A direct consequence of the first order conditions of the regulator's decision program (4) is the following proposition. ${ }^{8}$

Proposition 1 Under Assumption (A2), for a given quality level a, the price response correspon-

\footnotetext{
${ }^{7}$ For greater generality that will turn out to be needed for some of our results, we do not impose concavity assumptions on profits. Hence $p^{m}(a)$ may well be multi-valued (i.e., a correspondence).

${ }^{8}$ Throughout the paper, "increasing" will mean "weakly increasing". We will say that a correspondence is increasing if every one of its selections is (weakly) increasing.
} 
dence of the social planner is increasing in quality a. Indeed, we have

$$
p^{s}(a)=c(a) .
$$

A direct consequence of the fact that the social planner always sets price at marginal cost, so that the firm's profit is equal to zero at the social optimum, is that at a given quality level the monopoly price is necessarily larger than the socially optimal price.

Proposition 2 Under Assumptions (A1)-(A2), for any exogenously given quality level a, we have

$$
p^{m}(a) \geq p^{s}(a)
$$

In contrast, from the perspective of the monopoly firm, the dependence of the optimal price on exogenous quality is a significantly more complex issue, as we now see. To this end, we shall need the notion of (strict) log-supermodularity of demand, which is defined by the log of demand, or $\log D(p, a)$, being a (strictly) supermodular function of $(p, a)$, or using the cross partial test, $\frac{\partial^{2} \log D(p, a)}{\partial p \partial a}>0$, which boils down to

$$
D(p, a) D_{p a}(p, a)-D_{p}(p, a) D_{a}(p, a)>0 \text { for all }(p, a) .
$$

The assumption of log-supermodularity of demand is quite general, since we always have in (5) that $D_{p}(p, a) D_{a}(p, a) \leq 0$ under Assumption (A1). ${ }^{9}$ Thus the condition (5) imposes only that the demand function not be too submodular in $(p, a)$, or that the cross partial $\frac{\partial^{2} D(p, a)}{\partial p \partial a}$ not be too negative.

Importantly, this assumption has a very natural economic interpretation for the effect of quality on people's willingness to pay: Indeed, this property is precisely equivalent to assuming that higher quality strictly increases the price elasticity of demand. ${ }^{10}$ This constitutes a natural assumption, indeed even a precise definition, for the notion of product or environmental quality in the context at hand.

\footnotetext{
${ }^{9}$ By Topkis's (1978) cross partial test, log-supermodularity of demand, or supermodularity of log-demand, is equivalent to the familiar complementarity property that $\frac{\partial^{2} \log D(p, a)}{\partial p \partial a} \geq 0$, for all $(p, a)$, which is easily seen to correspond to $(5)$.

${ }^{10}$ Denoting by $\varepsilon(p, a)=\frac{p D_{p}(p, a)}{D(p, a)}$ the price elasticity of demand, one easily checks that

$$
\frac{\partial \varepsilon(p, a)}{\partial a}=\frac{p\left[D(p, a) D_{p a}(p, a)-D_{p}(p, a) D_{a}(p, a)\right]}{[D(p, a)]^{2}} .
$$

Hence, for $(p, a)$ such that $p>0$ and $D(p, a)>0$, condition (5) is equivalent to $\frac{\partial \varepsilon(p, a)}{\partial a}>0$.
} 
The next result provides two distinct but closely related sufficient conditions for the intuitive conclusion that the firm's price is increasing in its product quality.

Proposition 3 Assume that the demand function satisfies either one of the following two conditions

$$
\frac{c^{\prime}(a)}{[p-c(a)]^{2}}+D(p, a) D_{p a}(p, a)-D_{p}(p, a) D_{a}(p, a)>0 \text { for all }(p, a)
$$

or

$$
D_{a}(p, a)+[p-c(a)] D_{p a}(p, a)-c^{\prime}(a) D_{p}(p, a)>0 \text { for all }(p, a)
$$

Then the price response correspondence, $p^{m}(a)$, of the firm is globally increasing in quality a.

Each of the two sufficient conditions is easily seen to hold with great generality. Indeed, for (6), as the first term is always $\geq 0$, the conclusion may hold even when the log-supermodularity of demand (or condition (5)) fails. On the other hand, if one were to think of (6) as a condition on demand that guarantees that $p^{m}(a)$ is globally increasing for all possible quality cost functions, then the log-supermodularity of demand is exactly what is needed (this is easily seen to be the case with the limit case of a constant cost function, i.e., $c(a)=c$ for all $a)$.

Similarly, as to (7), the first and third terms are both always positive, so the only requirement is again that $D_{p a}(p, a)$ not be too negative. Thus, the two conditions in this Proposition are qualitatively very similar.

Thus, while price and quality are always complements at the social level, they are complements at the private level for a broad class of demand functions, but not quite a universal one. In other words, in first-best planning, a social planner will always price higher for better quality, while a monopoly firm will have a tendency to do so, but this is guaranteed only under the condition (6) or (7) and, in particular, under the intuitive assumption of log-supermodularity of demand, i.e., when the price elasticity of demand is strictly increasing in quality.

Going even further, we now see that, to some extent, a partial inversion of the arguments above may be used to provide sufficient conditions for the reverse -highly unusual-conclusion that price is globally decreasing in product quality. ${ }^{11}$

Proposition 4 Assume that the demand function satisfies the following condition

$$
D_{a}(p, a)+[p-c(a)] D_{p a}(p, a)-c^{\prime}(a) D_{p}(p, a)<0 \text { for all }(p, a) .
$$

\footnotetext{
${ }^{11}$ In contrast to the previous proposition, only one sufficient condition is given here, as condition (5) cannot be sign-inverted, due to the term $\frac{c^{\prime}(a)}{[p-c(a)]^{2}}$ being always $\geq 0$, and arbitrarily close to $+\infty$ as $p$ gets close to $c(a)$.
} 
Then the price response correspondence, $p^{m}(a)$, of the firm is globally decreasing in quality a.

The fact that a monopoly firm might set a high price for a low-quality product (or a brown good) and a lower price for a higher quality product (or a green good) is certainly somewhat provocative and very counter-intuitive from the standpoint of consumers. Indeed one often takes for granted that higher-quality products will be more expensive.

It is quite surprising for economists as well for two different reasons. The first is that most consumers exhibit a higher willingness to pay for greener products (as argued in the Introduction), and firms should be expected to therefore price green goods higher. The second reason is that higher quality is posited to be more costly for the firm to produce, which is clearly a very natural assumption in most settings, including the environmental case.

On the other hand, as should be expected, the above results are theoretically consistent with this possibility being relatively difficult to observe in practice, in the sense that (8) is an extremely restrictive condition on demand. It requires $D_{p a}(p, a)$ to be strongly negative, since the first and third terms in (8) are always positive.

In terms of finding actual explicit examples where price is not globally increasing in quality, one would not expect the search to be easy, given that most of the well known demand functions have the property that the price elasticity is strictly increasing in quality (see Vives, 1999), i.e., satisfy (5). ${ }^{12}$ It is of course easier to find examples where price decreases in quality locally than examples where this counter-intuitive property holds globally. Nevertheless, using a familiar class of demand functions, we provide a simple and plausible example where the latter, quite surprising, property holds.

\section{Example 3.1 :}

Consider a monopolist having demand and unit cost functions given by

$$
D(p, a)=\frac{1}{(p-a+1)^{2}}, p \geq 0, a \in[0,1] \text {, and } c(a)=\frac{a}{4} .
$$

It is easy to verify that this specification satisfies all our assumptions. The firm's profit function is

$$
\pi(p, a)=\left(p-\frac{a}{4}\right) \frac{1}{(p-a+1)^{2}}
$$

\footnotetext{
${ }^{12} \mathrm{~A}$ different sort of counter-intuitive internal substitutability between price and quality was obtained by Kim (2003) when a firm facing an increase in demand-related risk uses price and quality as alternative instruments to spread risk. This author proves that, under the condition that the expected utility function is submodular, when a firm facing demand uncertainty sets price and quality simultaneously, it responds to an increase in risk by reducing price and improving quality.
} 
The first order condition for the firm's choice of price, $\pi_{p}(p, a)=0$, reduces to $\frac{1-p-a / 2}{(p-a+1)^{3}}=0$ or

$$
p=1-\frac{a}{2}
$$

In addition the second order condition holds since $\pi_{p p}\left(1-\frac{a}{2}, a\right)=-\left(2-\frac{3}{2} a\right)^{3}<0$ for all $a \in[0,1]$.

Thus, by inspection of (9), the price charged by the monopoly firm is globally decreasing in quality. ${ }^{13}$ The highest optimal price will be charged for the lowest possible quality that the firm can produce!

As a preview of the situation where price and quality are simultaneously chosen, we now show that, in this example, the monopolist chooses the highest quality level, which is also socially optimal, and charges a price larger than the social planner's.

Indeed, denote by $\bar{\pi}(a)=\pi\left(1-\frac{a}{2}, a\right)$. One can check that $\bar{\pi}(a)=\frac{1-\frac{3}{4} a}{\left(2-\frac{3}{2} a\right)^{2}}$ and $\bar{\pi}^{\prime}(a)=$ $\frac{3\left(1-\frac{3}{4} a\right)}{2\left(2-\frac{3}{2} a\right)^{3}}>0$ for all $a \in[0,1]$. Hence,

$$
a^{m}=1 \text { and } p^{m}=\frac{1}{2}
$$

Total surplus is $W(p, a)=\frac{1}{p-a+1}+\left(p-\frac{a}{4}\right) \frac{1}{(p-a+1)^{2}}$. Solving the two first order conditions, it is easy to check that the social optimum calls for

$$
a^{s}=1 \text { and } p^{s}=\frac{1}{4}
$$

Thus the planner's optimal price is lower than the monopolist's, as expected since the corresponding optimal qualities are the same.

We conclude this section with two remarks.

Remark 1.We stress that while the optimal monopoly price may well decrease with quality, in a robust and even global sense, the restrictiveness of the underlying sufficient conditions suggests that this is not likely to be an empirically relevant (or widespread) phenomenon. On the other hand, even though it will tend to fail to hold globally, this unusual property may hold locally, and possibly at relevant parts of the quality range.

Remark 2. Since the socially optimal price always increases with quality (as seen above), the possible failure of this intuitive monotonicity property for the firm's price may be construed as yet another distortion due to monopoly pricing.

\footnotetext{
${ }^{13}$ Note that this result is obtained despite this example does not fulfill the condition (8).
} 


\subsection{The case of fixed price}

We now consider the reverse problem of monopoly choice of quality for a given fixed price

$$
\max \{[p-c(a)] D(p, a): a \in[0, A]\} .
$$

Such a situation might arise for instance if the monopolist is subject to government pricing regulation in its traditional role of price setter. The most obvious example would be a binding price ceiling (or price floor).

Before looking at the case of fixed price, it is instructive to first present the case of fixed quantity (or output) when the firm chooses quality. This scenario is actually of interest for this model, in view of the fact that Spence's pioneering paper reasoned in terms of this scenario in order to shed light on the overall properties of this model, as we now briefly review.

Spence argued that, in addition to the usual mark-up above the marginal cost of production, the firm might also set quality too high or too low relative to the social optimum. This second inefficiency result is better understood for the firm's decision problem written in terms of quantity (instead of price) and quality. Denote by $P(x, q)$ the inverse demand where $x$ is the quantity produced by the firm. The social welfare can be written as a function of the quantity-quality pair,

$$
\widetilde{W}(x, a)=\int_{0}^{x} P(t, a) d t-x P(x, a)+\pi(x, a) .
$$

Therefore, the marginal impact of an increase in quality on social welfare is

$$
\frac{\partial \widetilde{W}}{\partial a}(x, a)=\int_{0}^{x} P_{a}(t, a) d t-x P_{a}(x, a)+\frac{\partial \pi(x, a)}{\partial a} .
$$

Setting $\frac{\partial \pi}{\partial a}=0$, the sign of $\frac{\partial \widetilde{W}}{\partial a}$ depends on the impact of quality on consumer surplus which, in turn, depends on the relative magnitudes of the average valuation of quality increments over all the people in the market, $\frac{1}{x} \int_{0}^{x} P_{a}(t, a) d t$, and the marginal valuation of quality increments by the marginal consumer, $P_{a}(x, a)$. When the average valuation of quality increments exceeds the marginal valuation, the firm sets quality too low; it stops increasing quality too soon. This relative magnitude is well known to rely on the sign of the second order cross derivative $P_{x a}$. This forms in fact the main result of [Spence, 1975, Proposition 1].

Proposition 5 (i) For given $x$, the firm undersupplies (oversupplies) quality relative to the social optimum when

$$
\frac{1}{x} \int_{0}^{x} P_{a}(t, a) d t>(<) P_{a}(x, a) .
$$

(ii) For all $(x, a)$, a sufficient condition for (12) is that $P_{x a}(x, a)<(>) 0$. 
As pointed out by Varian (1992), Proposition 5 is easily understood graphically. An increase in quality generates two effects on the demand curve. First, the demand curve shifts up. Second, the inverse demand curve becomes flatter (respectively steeper) if $P_{x a}<0$ (resp., $P_{x a}>0$ ). In this case, for a fixed production level, the monopolist undersupplies (resp. oversupplies) quality relative to the social optimum.

These rotations of the demand curve stem from changes in the dispersion of consumers' valuations. More precisely, an increase in the dispersion of consumers' valuations rotates the demand curve clockwise $\left(P_{x a}>0\right)$. The impact of these clockwise rotations on the monopolist's behavior has been thoroughly studied by Johnson and Myatt (2016). They prove that in many settings, profits are $U$-shaped functions of the dispersion. High dispersion is complemented by niche production and low dispersion is complemented by mass-market supply.

On the other hand, we shall now see that in price-quality space, a clear-cut result on quality choice obtains when the price is held fixed, or taken as given, as in problem (10). Beforehand, we warn the reader that, in the present context, reasoning on the basis of a fixed price is not equivalent to reasoning on the basis of a fixed output. Indeed, with price held fixed, the impact of quality on consumer surplus is no longer ambiguous since it relies on the impact of quality on market demand which is strictly positive. Hence, with price held fixed, the firm always sets quality lower relative to a social planner, since

$$
\frac{\partial W}{\partial a}(p, a)=\int_{p}^{+\infty} D_{a}(t, a) d t+\frac{\partial \pi(x, a)}{\partial a}
$$

and thus, when $\frac{\partial \pi}{\partial a}=0$, we have $\frac{\partial W}{\partial a}>0$. This proves the following useful observation.

Proposition 6 For any given fixed price $p$, the firm always undersupplies quality relative to the social optimum.

It is important to stress that, unlike the case of fixed output level, at fixed price, the firm does undersupply quality relative to the social optimum. Nevertheless, this does not imply that if one compares the social optimum in both price and quality and the monopolist's solution pair, that one will always find quality lower in the latter.

It is the tendency for a low quality reflected in this result that has led regulatory authorities to specify minimum quality standards in the context of price regulation. Spence (1975) and Sheshinski (1976) discuss some aspects of this type of regulation of a monopoly firm in some detail.

As a final question in this subsection, we look at the problem of whether a price-constrained firm (through regulation) will choose a higher or lower quality as the price it is forced to charge 
increases. This is obviously the reverse question from the one addressed in the previous subsection. It turns out that this problem satisfies analogous properties as the reverse one.

Proposition 7 (i) Assume that the demand function satisfies either the condition (6) or (7). Then the quality response correspondence, $a^{m}(p)$, of the firm is globally increasing in price $p$.

(ii) Assume that the demand function satisfies condition (8). Then $a^{m}(p)$ is globally decreasing in price $p$.

Indeed, since the condition of log supermodularity of demand treats the price and quality variables symmetrically, Propositions 3 and 4 also imply that, treating price as a parameter and quality as the decision variable, the monopolist will choose an optimal quality that is increasing in price if condition (6) or (7) holds, but instead decreasing in the price if condition (8) holds. Naturally, the more intuitive situation is the one where a higher quality is associated with a higher price limit, and this is the scenario that is predicated on a very general assumption on the demand function, just as in the reverse problem.

Interestingly, a dual economic interpretation of the condition of log supermodularity of demand holds here: It says that the quality elasticity of demand, defined by $\widehat{\varepsilon}(p, a)=\frac{a D_{a}(p, a)}{D(p, a)}$, increases in the regulatory price. ${ }^{14}$ In other words, demand increases more (in percentage terms) in response to a quality increase when the price imposed on the firm is higher.

While the situation of a fixed price is less economically relevant than the case where quality is the fixed variable, it is worth noting that the above analysis applies equally to both cases. In addition, this result sheds some new light on the second market failure (in quality choice) of the monopoly regime with endogenous quality.

\section{A microeconomic foundation}

In this section, we offer a simple micro-economic foundation for the demand function at hand, when the utility function depends directly on product quality. In so doing, we build on Ebert and von dem Hagen (1998) and the main goal is to derive a sufficient condition on the utility function

\footnotetext{
${ }^{14}$ Indeed, one easily checks that

$$
\frac{\partial \widehat{\varepsilon}(p, a)}{\partial p}=\frac{a\left[D(p, a) D_{p a}(p, a)-D_{p}(p, a) D_{a}(p, a)\right]}{[D(p, a)]^{2}} .
$$

Hence, for $(p, a)$ such that $p>0$ and $D(p, a)>0$, condition $(5)$ is equivalent to $\frac{\partial \widehat{\varepsilon}(p, a)}{\partial p}>0$.
} 
that yields a direct demand function satisfying the key property of log-supermodularity in price and quality (i.e., condition (5) here).

Let us suppose that the market demand curve is generated from a representative consumer with income $w$ by maximizing a quasi-linear utility function $U(x, a)+y$, where $x$ stands for the quantity of the good under consideration (with price $p$ ), and $y$ is the numeraire good (with price normalized to 1$)$.

The consumer problem is then

$$
\max _{x, y \geq 0} U(x, a)+y
$$

subject to

$$
p x+y=w .
$$

We make the natural assumption that

(A3) $U$ is three times continuously differentiable and satisfies $U_{x} \geq 0, U_{a} \geq 0, U_{x x} \leq 0$, $\lim _{x \rightarrow 0} U_{x}(x, a)=+\infty$ and $\lim _{x \rightarrow+\infty} U_{x}(x, a)=0$.

Solving the constraint to get $y=w-p x$, and substituting into the utility, the consumer problem is equivalent to (the unconstrained problem)

$$
\max _{x \geq 0} U(x, a)+w-p x
$$

Proposition 8 Under Assumption (A3),

(i) $D(p, a)$ is decreasing in $p$.

(ii) $D(p, a)$ is strictly increasing in a if $U_{x a}>0$ for all $(x, a)$.

(iii) $D(p, a)$ is log-supermodular in $(p, a)$ if $U$ satisfies,

$$
U_{x x} U_{x a}+x\left(U_{x x x} U_{x a}-U_{x x a} U_{x x}\right)<0
$$

for all $(x, a)$.

The Law of Demand at the individual level follows here from the fact that, for a quasi-linear utility function, the income effect induced by a price change vanishes. Therefore, the price effect reduces to the substitution effect, which is well known from standard consumer theory to be negative. ${ }^{15}$

\footnotetext{
${ }^{15}$ This framework allows for a consumption sector where households have heterogenous income. Nevertheless, since individual demands are income independent, $D(p, a)$ denotes both the individual demand and the mean demand in the population.
} 
The meaning of condition (13) directly on the utility function is as follows. It holds if and only if marginal utility $U_{x}$ satisfies the gross complements condition from standard consumer theory (see e.g., Amir and Bloch, 2009 for an overview) with respect to quantity and quality. ${ }^{16}$ We stress that this condition is more restrictive than usual since it is the marginal utility function that must satisfy it, and not the utility function itself. At the same time, the condition actually holds quite broadly. Indeed, among the commonly used utility functions, it is satisfied, among many others, by the quadratic utility, the Cobb-Douglas utility, the entire class of multiplicatively separable utility of the form $U(x, a)=g(a) u(x)$, with $g^{\prime}>0, u^{\prime}<0, u^{\prime \prime}<0$, if $u$ is log-concave (i.e., if $\left.u^{\prime} u^{\prime \prime \prime}-\left(u^{\prime \prime}\right)^{2} \leq 0\right)$. The latter is quite a general class of utility functions. As examples of this class, we have the subclass of CARA utility of the form $U(x, a)=g(a) e^{-x}$, with $g^{\prime}>0$, among others.

As to a precise interpretation for condition (13), it is obviously not so transparent to reason in terms of the third derivatives of the utility function. Instead, we prefer to go back to the clear and natural equivalent economic interpretation in terms of the demand function having a price elasticity that increases with the quality level. Again, the latter can clearly serve as a very definition of product quality, in particular in an environmental setting.

\section{$5 \quad$ Setting both price and quality}

In the general monopoly case with the two inter-related variables, the relationship between the (first-best) optimal and profit-maximizing quality levels is determined by the interaction of the extent to which the monopolist sets a price larger than its marginal cost of production and the effect of the second market failure induced by the choice of product quality. In this section, we point out that due to this new factor of inefficiency, the usual inefficiency result might go in the opposite direction : The monopolist might set a price lower than the social optimum. We give an example of a monopoly regime where this quite surprising result obtains. No such example has been provided in the literature.

For the most empirically relevant case ${ }^{17}$, according to section 3 , where price and quality are

\footnotetext{
${ }^{16}$ For extra clarity here, we recall some standard facts from classical consumer theory for the two-good case (say $x$ and $y)$.

(i) two goods $x$ and $y$ are gross complements if the demand for good $x$ decreases with the price of good $y$.

(ii) a sufficient condition for $x$ and $y$ to be gross complements is that the utility function $V(x, y)$ satisfies the gross complements condition, which is $V_{x} V_{y}+x\left(V_{x x} V_{y}-V_{x y} V_{x}\right)<0$ for all $(x, y)$.

${ }^{17}$ This case is obtained in our framework if the demand function satisfies the condition (6) or (7) while it is obtained in Sheshinsky (1976) under more stringent conditions, including a concavity assumption of the profit function.
} 
strategic complements for both the regulator and the monopoly firm, Sheshinsky (1976) has established that, if $P_{x a}<0$ (hence for a fixed price the monopolist undersupplies quality), one can exclude only the possibility that

$$
p^{m}<p^{S} \text { and } a^{m}>a^{S} .
$$

All the other three possible rankings of the two price-quantity pairs $(p, a)$ chosen by the firm and the planner remain possible, i.e., are consistent with our assumptions (and those of Spence, 1975 and Sheshinski's, 1976). A noteworthy implication of (14) is that a necessary condition for the monopolist to choose a price lower than the social planner's is that the firm also undersupplies quality. ${ }^{18}$

In addition, the quality distortion between the level chosen by the monopolist and the social optimum is influenced by traditional mark-up effects and therefore by the price-elasticity of demand. In particular, Spence (1975) has established that if the price elasticity is independent of the price and strictly increases with quality, then quality is undersupplied by the monopolist. We build on this result and show that the monopolist then chooses a price lower than the social planner. Furthermore, we derive a necessary and sufficient condition for the monopolist to choose a price lower than the social optimum for the class of demand functions with price-elasticity independent of price. Finally, we provide specific functional forms to the demand and the cost functions such that the closed-form solutions have the monopolist pricing lower than the social optimum.

To investigate this, let

$$
\bar{\pi}(a)=\max _{p} \pi(p, a) \text { and } \bar{W}(a)=\max _{p} W(p, a) .
$$

When the monopolist's quality choice $a^{m}$ is interior, i.e. $0<a^{m}<+\infty$, as pointed out by Spence (1975) the question of whether the firm under or oversupplies quality relative to the social optimum translates formally into the question of whether the derivative $\bar{W}^{\prime}\left(a^{m}\right)$ is strictly positive or strictly negative. This question can be raised for any demand function, but the case in which the elasticity is independent of price is illuminating.

\subsection{Price-independent demand elasticity}

In this section, we suppose that the demand function is of the constant elasticity type for any given level of quality, so that $D(p, a)=g(a) p^{\varepsilon(a)}$ where $g: \mathbb{R}_{+} \rightarrow \mathbb{R}_{+}$is an increasing function and

\footnotetext{
${ }^{18}$ One might be tempted to conclude from Proposition 5 that if $P_{x a}=0$ (i.e., if is additively separable in $x$ and $a$ ), then the firm will choose the socially optimal price. However, this is correct at fixed output $x$, but not necessarily if output is also chosen by the firm and the planner.
} 
$\varepsilon(a)<-1$ for all $a,{ }^{19}$ and $\varepsilon(a)$ represents the price elasticity of demand, i.e. $\varepsilon(a)=\frac{D_{p}(p, a) p}{D(p, a)}$. For this class of demand functions, we can establish the following proposition.

Proposition 9 Under the assumption that the monopolist chooses an interior quality level, i.e. when $\bar{\pi}^{\prime}\left(a^{m}\right)=0$ with $0<a^{m}<1$, if the price elasticity

(i) strictly increases with quality, i.e., $\varepsilon^{\prime}\left(a^{m}\right)>0$, then $\bar{W}^{\prime}\left(a^{m}\right)>0$, so quality is undersupplied by the monopolist, i.e. $a^{m}<a^{s}$.

(ii) strictly declines with quality, i.e., $\varepsilon^{\prime}\left(a^{m}\right)<0$, then $\bar{W}^{\prime}\left(a^{m}\right)<0$, so quality is oversupplied by the monopolist, i.e. $a^{m}>a^{s}$.

(iii) does not vary with quality, i.e., $\varepsilon^{\prime}\left(a^{m}\right)=0$, then $\bar{W}^{\prime}\left(a^{m}\right)=0$, so quality is set at the optimal level by the monopolist, i.e. $a^{m}=a^{s}$.

This corresponds to Proposition 3 in Spence (1975) with the correction that the conclusions are only valid under the additional assumption that the quality level chosen by the monopolist is interior, namely neither minimal nor maximal. The necessity of this additional assumption is illustrated in Appendix 8.7 by an example where despite the fact that price elasticity strictly increases with quality, the quality is set at the socially optimal level by the monopolist (in apparent contradiction of the above Proposition). This comes from the fact that the monopolist chooses there the maximal quality level, which is also socially optimal.

For the current class of demand functions, we shall now establish a necessary and sufficient condition for the very counter intuitive result that the monopoly firm charges a price lower than the social optimum.

Proposition 10 The monopoly firm charges a price lower than the social planner's if and only if

$$
\frac{\varepsilon\left(a^{m}\right)}{\varepsilon\left(a^{m}\right)+1}<\frac{c\left(a^{s}\right)}{c\left(a^{m}\right)}
$$

To shed some light on this result, note that the function $\varepsilon \mapsto \frac{\varepsilon}{\varepsilon+1}$ is increasing over $(-\infty,-1)$ and takes values in $(1,+\infty)$ with $\lim _{\varepsilon \mapsto-\infty} \frac{\varepsilon}{\varepsilon+1}=1$. Hence, the necessary and sufficient condition (15) is more likely to hold if the price elasticity is low. If one considers the case where the price elasticity strictly increases with quality, as suggested in the introduction of this section, then from Proposition 9 we know that the monopolist undersupplies quality, i.e. $a^{m}<a^{s}$ and, i.e. $1<\frac{c\left(a^{s}\right)}{c\left(a^{m}\right)}$. To illustrate that condition (15) may well hold in a meaningful setting, we provide an explicit example with closed form solutions.

\footnotetext{
${ }^{19}$ It is well known that the monopolist operates on the elastic part of the demand curve.
} 


\subsection{A first example where the monopoly price is lower than the planner's}

In this subsection, we give functional forms to the elasticity of demand and to the marginal cost of production in order to prove that the class of models fulfilling condition (15) is not empty. Consider the specification

$$
\left\{\begin{array}{c}
D(p, a)=p^{-\left(1+\frac{1}{a}\right)} \\
c(a)=a^{2}
\end{array}\right.
$$

i.e. $g(a)=1$ and $\varepsilon(a)=-\left(1+\frac{1}{a}\right)$. In addition, we assume that the quality level $a \in\left[a_{\min }, a_{\max }\right]$ with $1 \leq a_{\min }$ and $2 \leq a_{\max } \leq 3$. The profit function is then

$$
\pi(p, a)=\left(p-a^{2}\right) p^{-\left(1+\frac{1}{a}\right)} .
$$

As all prices $p<1$ are dominated choices (since $\pi(p, a)<0$ ), we can focus on prices larger than one. For all $(p, a) \in[1,+\infty) \times\left[a_{\min }, a_{\max }\right]$, Assumptions (A1) and (A2) hold. ${ }^{20}$

For a given quality level, it can be verified that the price set by the monopoly firm is

$$
p^{m}(a)=(1+a) a^{2}
$$

while the regulator charges

$$
p^{s}(a)=a^{2} .
$$

Here, price and quality are strategic complements for the monopoly firm since condition (6) holds. ${ }^{21}$ In addition, one easily checks that $P_{x a}(x, a)<0$ for all $(x, a) \in[0,1] \times\left[a_{\min }, a_{\max }\right] .{ }^{22}$ In this case, we know from Sheshinsky (1976) that, if the monopolist sets a price lower than the social optimum,

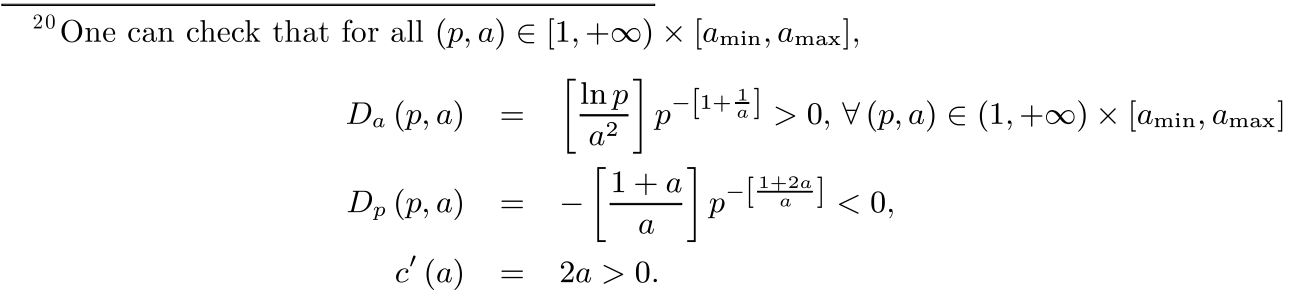

${ }^{21}$ One can check that

$$
\frac{c^{\prime}(a)}{[p-c(a)]^{2}}+D(p, a) D_{p a}(p, a)-D_{p}(p, a) D_{a}(p, a)=\frac{2 a}{[p-2 a]^{2}}>0, \forall(p, a) .
$$

${ }^{22}$ Effectively, we get

$$
P_{x a}(x, a)=\frac{1}{[1+a]^{2}} x^{-\frac{[1+2 a]}{[1+a]}}\left[-1+\left[\frac{a}{1+a}\right] \ln x\right]<0
$$

for all $(x, a) \in[0,1] \times\left[a_{\min }, a_{\max }\right]$. 
he cannot oversupply quality. In this example, the monopolist does set a price lower than the social optimum for a lower quality level. We get,

$$
\left\{\begin{array}{c}
\bar{\pi}(a)=a^{\frac{a-2}{a}}(1+a)^{-\frac{(1+a)}{a}} \\
\bar{W}(a)=a^{\frac{a-2}{a}}
\end{array} .\right.
$$

These two functions are convex over $[1,3]$ and thus admit corner maxima. By comparing their corner values, one can find $a_{\min }$ and $a_{\max }$ such that $1 \leq a_{\min }<1.5$ and $2 \leq a_{\max } \leq 3$ and the monopolist chooses the lowest quality level, i.e. $\bar{\pi}\left(a_{\min }\right)>\bar{\pi}\left(a_{\max }\right)$. Yet the largest quality level is socially optimal, i.e. $\bar{W}\left(a_{\max }\right)>\bar{W}\left(a_{\min }\right)$, and condition (15) holds. Take as an illustration $a_{\min }=1$ and $a_{\max }=3$, we get

$$
\left\{\begin{array}{l}
a^{m}=1<a^{s}=3 \\
p^{m}=2<p^{s}=9
\end{array} .\right.
$$

\section{The Spence model as a one-dimensional problem}

In this section, we uncover an interesting property of the Spence model, namely that under some reasonable specification of the demand and quality cost functions, the problems of determining the optimal price-quality pair by both the firm and the planner collapse to a one-dimensional problem each, consisting of choosing a single composite variable of price and quality.

We then invoke the same construction to provide a second specific example to demonstrate that when both prices and qualities are chosen, out of the continuum of possible optimal choices, the monopolist's price may well be selected to be lower than the social planner's price.

\subsection{The one-dimensional problem}

Consider the class of demand functions

$$
D(p, a)=\frac{\lambda c(a)}{p^{2}}
$$

where $\lambda>0$. This is a specific example of the model with price-independent demand elasticity with $g(a)=\lambda c(a)$ and $\varepsilon(a)=-2$. In this case, Proposition 5 establishes that for a given output level, the quality choice of the monopolist is socially optimal. Nevertheless, we shall point out in this section that, for this class of demands, there is an indeterminacy of the optimal price-quality pair.

The profit function is

$$
\pi(p, a)=[p-c(a)] \frac{\lambda c(a)}{p^{2}}
$$


The first-order conditions w.r.t. $p$ and $a$ are respectively

$$
\left\{\begin{array}{c}
\frac{\lambda c(a)}{p^{2}}-2[p-c(a)] \frac{\lambda c(a)}{p^{3}}=0 \\
-\frac{\lambda c(a)}{p^{2}} c^{\prime}(a)+[p-c(a)] \frac{\lambda c^{\prime}(a)}{p^{2}}=0
\end{array} .\right.
$$

One easily checks that both these first-order conditions reduce to the same relationship

$$
p^{m}=2 c\left(a^{m}\right) .
$$

Therefore, the monopoly firm chooses any price-quality pair $\left(p^{m}, a^{m}\right)$ such that (17) holds.

The social welfare function reduces to

$$
W(p, a)=\frac{\lambda c(a)}{p}+[p-c(a)] \frac{\lambda c(a)}{p^{2}} .
$$

Similarly, the social optima, $\left(p^{s}, a^{s}\right)$, are again characterized by two first-order conditions, which reduce to the same relationship ${ }^{23}$

$$
p^{s}=c\left(a^{s}\right)
$$

In other words, for each of the two problems, there is a continuum of solutions, specified by one single first-order condition (the condition (17) for the firm and the condition (18) for the social planner) involving both choice variables. Put differently, for each problem, the two associated first-order conditions simply coincide.

To restate the problems in their one-dimensional versions, with decision variable $\frac{c(a)}{p}$, one can rewrite the profit and welfare functions as

$$
\pi(p, a)=\lambda\left[1-\frac{c(a)}{p}\right] \frac{c(a)}{p}
$$

and

$$
W(p, a)=\lambda \frac{c(a)}{p}+\left[1-\frac{c(a)}{p}\right] \frac{\lambda c(a)}{p} .
$$

This form makes it is transparent that both objectives are functions of only one variable, $\frac{c(a)}{p}$, which may be viewed as representing price-adjusted quality. Alternatively, one may express both functions in terms of the single variable $\frac{p}{c(a)}$, which then stands for quality-adjusted price. Both these ratios are natural economic variables.

With the latter interpretation, (17) and (18) hold that the firm and the social planner will choose unique optimal quality-adjusted prices of 2 and 1 respectively. However, each of these solutions corresponds to a continuum of possible optimal price-quality pairs for the original model.

\footnotetext{
${ }^{23}$ Note that this is no longer the case if $\varepsilon(a) \neq-2$.
} 
This suggests that, for this class of demand specifications, the Spence model is indeterminate, and naturally collapses into a well-defined one-dimensional problem consisting of choosing a qualityadjusted price. In this form, one recovers the intuitive result that the social planner will always charge a lower quality-adjusted price than a monopolist.

We close this discussion with one remark linking the present quality-dependent demand to the theory of vertical product differentiation. Dealing with tractable forms of demand functions for vertically differentiated products, Sutton $(1991,1998)$ proposes several functional forms each of which is a function of a single variable, a quality-adjusted price (or a quality-adjusted output). The class of demand functions considered in this subsection may then be seen as providing a direct link between Spence's notion of product quality and Sutton's formulations. In other words, the present class of demand constitutes a theoretical foundation for Sutton's tractable model of vertical product differentiation.

\subsection{A second example where the monopoly price is lower than the planner's}

In this subsection, the previous specification is used to argue that it is possible for the firm to price lower than the social planner when quality is endogenous for each of the two problems, provided that, at the same time, the firm selects a lower quality than the social planner.

Due to the indeterminacy of the private optimum and of the social optimum, as reflected in the respective first-order conditions

$$
p^{m}=2 c\left(a^{m}\right) \text { and } p^{s}=c\left(a^{s}\right)
$$

the monopoly firm might well choose to charge a price $p^{m}$ lower than the social optimum $p^{s}$, as long as the corresponding qualities are taken from (17) and (18) respectively.

For illustrative purposes, we provide two specific examples:

(i) If $c(a)=a$, then (17) and (18) reduce to $p^{m}=2 a^{m}$ and $p^{s}=a^{s}$. For instance, one can then choose $p^{m}=10$ and $a^{m}=5$, together with $p^{s}=a^{s}=100$.

(ii) If $c(a)=a^{2}$, then (17) and (18) reduce to $p^{m}=2\left(a^{m}\right)^{2}$ and $p^{s}=\left(a^{s}\right)^{2}$. For instance, one can then choose $p^{m}=32$ and $a^{m}=4$, together with $p^{s}=100$ and $a^{s}=10$.

As reflected in the two examples, if one selects price solutions for the two problems such that $p^{m}<p^{s}$, then the monopoly firm must at same time choose a lower quality for the product than the planner, since

$$
p^{m}<p^{s} \Leftrightarrow 2 c\left(a^{m}\right)<c\left(a^{s}\right) \Longrightarrow c\left(a^{m}\right)<c\left(a^{s}\right) / 2<c\left(a^{s}\right) \Longrightarrow a^{m}<a^{s} .
$$


In conclusion, for this class of problems, there is a continuum of solution pairs at which the monopolist chooses a lower price and a lower quality than the social planner. Contrary to conventional wisdom, a monopolist may price lower than a social planner, but only when selling a lower quality variant. In case of a binary quality choice in an environmental setting, this might take the form of a monopolist choosing the brown variety and the social planner opting instead for the green variety, coupled with the unusual fact that the former is then priced lower (by the monopolist) than the latter (by the planner).

\section{Regulation via taxation or subsidization}

In this section, we review some results on optimal regulation via taxation or subsidization that were obtained by Ebert and Von dem Hagen (1998), and modify them appropriately for our setting. Since the modifications are minor, we shall simply describe the results and leave out the analytical arguments, due to their close proximity to those of Ebert and Von dem Hagen (1998).

In section 5, we pointed out that, when the monopolist sets both price and environmental quality, the only scenario that can be ruled out is where the firm sets a lower price than the social planner for a larger environmental quality. All other scenarios might emerge (lower price for lower quality, larger price for larger quality and larger price for lower quality). Nevertheless, by introducing two policy instruments, one can prove following Ebert and Von dem Hagen (1998) that the first-best allocation is obtained by always subsidizing consumption while environmental quality is either subsidized or taxed. In our framework, the purpose of the social planner is to maximize social welfare defined by (3). In other words, no additional social benefit of environmental quality is introduced. Here, we follow the literature in environmental economics where a damage function of pollution is introduced only when the utility of the representative consumer is separable in consumption and pollution, so that demand for the consumption commodity is independent of pollution. In our framework, the underlying utility function of the representative consumer (see section 4) is nonseparable in consumption and environmental quality, therefore market demand depends on environmental quality and therefore already takes into account the social benefit of environmental quality. The commodity tax corrects the usual market failure induced by the mark-up above marginal cost of production : One has to subsidize the monopolist in order to reach the socially optimal level of production. On the other hand, the Pigouvian tax on environmental quality has to take into account two opposite effects of environmental quality. As pointed out by Spence (1975) and recalled in subsection 3.2, 
for a given price level, the monopolist always undersupplies environmental quality with respect to the social optimum, but there is also an indirect effect of environmental quality due to the revision of the price strategy. The conclusion of our set-up is that, to solve the market failure generated by the monopolist's choice of environmental quality, if the marginal impact of quality increments on the marginal cost of production is larger (lower) than the marginal consumer's valuation of quality increments, one has to subsidize ( $\operatorname{tax}$ ) the monopolist. In other words, under this condition (the reverse condition) the second inefficiency induced by the choice of the environmental quality increases (decreases) the usual welfare loss of the monopoly.

In addition, in contrast to the well known result in the literature on environmental regulation under imperfect competition, that the Pigouvian tax should be lower than marginal damage (in the case of a negative externality) or equivalently that the Pigouvian subsidy should be larger than the marginal benefit, here the Pigouvian subsidy on environmental quality is always lower than marginal benefit. This reflects a complementarity between the environmental quality and the consumption level. The subsidy gives the firm the incentive to increase the environmental quality of its product. Due to the complementarity between quality and the consumption level, consumers increase their demand. Therefore, the Pigouvian subsidy required to reach the first-best optimum is lower than the social marginal benefit of environmental quality. In Ebert and Von dem Hagen (1998) the utility of the representative consumer depends on the consumption of a commodity and pollution emissions in a nonseparable way. In this case of pollution emissions, the commodity and emissions could either be complements or substitutes (hence, the Pigouvian tax could either be larger or lower than the marginal damage).

Finally, even if the marginal social benefit of quality increments in the optimal allocation equals zero, two instruments are needed to attain a first-best optimum and environmental quality is taxed.

\section{Appendix}

Here, we provide the proofs of the results that were not provided in the text, as well as an example where the monopoly choices coincide with the socially optimum. 


\subsection{Proof of Proposition 2}

From (4), we know that $\forall a, p^{s}(a)=c(a)$. At a given quality level $a$, the monopoly firm chooses $p^{m}(a)$ such that

$$
\pi\left(p^{m}(a), a\right) \geq 0 \Leftrightarrow\left[p^{m}(a)-c(a)\right] D\left(p^{m}(a), a\right) \geq 0 .
$$

Hence,

$$
p^{m}(a) \geq c(a)=p^{s}(a) .
$$

\subsection{Proof of Proposition 3}

Taking the $\log$ of the profit function, we have $\log \pi(p, a)=\log (p-c(a))+\log D(p, a)$. Hence, if (6) holds, we have

$$
\frac{\partial^{2} \log \pi(p, a)}{\partial p \partial a}=\frac{c^{\prime}(a)}{[p-c(a)]^{2}}+\frac{D(p, a) D_{p a}(p, a)-D_{p}(p, a) D_{a}(p, a)}{D^{2}(p, a)}>0 .
$$

The conclusion then follows from Topkis's Theorem applied to $\max \{\log \pi(p, a): p \in[c(a),+\infty)\}$, where $c(a)$ is increasing in $a$.

If instead (7) holds, then

$$
\frac{\partial^{2} \pi(p, a)}{\partial p \partial a}=D_{a}(p, a)+[p-c(a)] D_{p a}(p, a)-c^{\prime}(a) D_{p}(p, a)>0 \text { for all }(p, a) .
$$

The conclusion then follows from Topkis's Theorem applied to $\max \{\pi(p, a): p \geq 0\}$.

\subsection{Proof of Proposition 4}

From (8), it is easy to see that we now have instead

$$
\frac{\partial^{2} \pi(p, a)}{\partial p \partial a}=D_{a}(p, a)+[p-c(a)] D_{p a}(p, a)-c^{\prime}(a) D_{p}(p, a)<0 \text { for all }(p, a) .
$$

The conclusion then follows from the dual of Topkis's Theorem applied to $\max \{\pi(p, a): p \geq 0\}$.

\subsection{Proof of Proposition 8}

The consumer's demand $D(p, a)$ fulfills the familiar first order condition

$$
U_{x}(D(p, a), a)=p
$$


By differentiating the FOC with respect to $p$, then w.r.t. to $a$ and then twice w.r.t. to $p$ respectively, we get

$$
\left\{\begin{array}{c}
U_{x x} D_{p}=1 \\
U_{x x} D_{a}+U_{x a}=0 \\
\left(U_{x x x} D_{a}+U_{x x a}\right) D_{p}+U_{x x} D_{p a}=0
\end{array}\right.
$$

Using these conditions to solve for $D_{p}, D_{a}$ and $D_{p a}$, one easily gets parts (i) and (ii), and that

$$
D D_{p a}-D_{p} D_{a}>0 \Leftrightarrow \frac{1}{U_{x x}^{3}}\left[x U_{x x x} U_{x a}-x U_{x x a} U_{x x}+U_{x a} U_{x x}\right]>0 .
$$

Since we assumed that $U_{x x} \leq 0$, we get the desired inequality in part (iii).

\subsection{Proof of Proposition 9}

For a given quality level $a$, the interior price defined by (2) is a solution for the monopolist since the second order condition $\pi_{p p}\left(\frac{\varepsilon(a)}{\varepsilon(a)+1} c(a), a\right)=[\varepsilon(a)+1]\left[\frac{\varepsilon(a) c(a)}{\varepsilon(a)+1}\right]^{\varepsilon(a)-1} g(a)<0$ is fulfilled. Hence, the price set by the monopolist is

$$
p^{m}(a)=\frac{\varepsilon(a)}{[\varepsilon(a)+1]} c(a)
$$

In an analogous way, for a given quality level $a$, the interior price defined by (4) is a solution for the social planner since the second order condition $W_{p p}(c(a), a)=\varepsilon(a) g(a)[c(a)]^{\varepsilon(a)-1}<0$ is fulfilled. Hence, the regulator charges as always its marginal cost, i.e.

$$
p^{s}(a)=c(a)
$$

Then, one easily checks that

$$
\left\{\begin{array}{l}
\bar{\pi}(a)=\frac{[-\varepsilon(a)-1]^{-\varepsilon(a)-1} g(a)}{[-\varepsilon(a)]^{\varepsilon(a)} c(a)^{-\varepsilon(a)-1}} \\
\bar{W}(a)=\frac{g(a)}{[-\varepsilon(a)-1] c(a)^{-\varepsilon(a)-1}}
\end{array} .\right.
$$

This implies that

$$
\bar{W}(a)=\left[\frac{\varepsilon(a)}{\varepsilon(a)+1}\right]^{-\varepsilon(a)} \bar{\pi}(a) .
$$

For $0<a^{m}<A$ such that $\bar{\pi}^{\prime}\left(a^{m}\right)=0$, we get

$$
\bar{W}^{\prime}\left(a^{m}\right)=\varepsilon^{\prime}\left(a^{m}\right) \bar{\pi}\left(a^{m}\right)\left[\frac{\varepsilon\left(a^{m}\right)}{\varepsilon\left(a^{m}\right)+1}\right]^{-\varepsilon\left(a^{m}\right)}\left(-\ln \left[\frac{\varepsilon\left(a^{m}\right)}{\varepsilon\left(a^{m}\right)+1}\right]-\frac{1}{\left[\varepsilon\left(a^{m}\right)+1\right]}\right) .
$$

Since $-\ln \left(\frac{\varepsilon}{\varepsilon+1}\right)-\frac{1}{\varepsilon+1}>0$ for all $\varepsilon<-1$, the sign of the derivative $\bar{W}^{\prime}\left(a^{m}\right)$ is the sign of the derivative $\varepsilon^{\prime}\left(a^{m}\right)$. This proves (i), (ii) and (iii). 


\subsection{Proof of Proposition 10}

The condition follows directly from (20) and (21).

\subsection{When the monopolist's quality is socially optimal}

In this last part, we identify one circumstance for market demand not considered by Spence (1975) under which quality is set at the optimal level by the monopolist. This is a specific case of the model where the price elasticity of demand is independent of price.

Consider the Spence model of section 5.1 with the assumption that the minimal quality level is non-zero, i.e. $a \in\left[a_{\min }, a_{\max }\right]$ with $a_{\min }>0$. We shall assume in addition, as in subsection 5.2 that the price elasticity of demand strictly increases with quality. More precisely, let demand be

$$
D(p, a)=p^{-\left(1+\frac{1}{a}\right)} .
$$

In that case, Proposition 3 of Spence (1975) establishes that the monopolist undersupplies quality. Nevertheless, this result relies on the assumption (not mentioned) that the quality level chosen by the monopolist is unbounded. If the quality chosen by the monopolist cannot be arbitrarily large and he chooses the maximum quality level, so will the social planner. This is the case, in particular, when the marginal cost of production is of the exponential type, i.e.

$$
c(a)=e^{a} .
$$

In this case, for a given quality level, by substituting the functional forms into (20) it can be verified that the price set by the monopolist is

$$
p^{m}(a)=[1+a] e^{a},
$$

while the regulator charges

$$
p^{s}(a)=e^{a}
$$

This yields

$$
\left\{\begin{array}{c}
\bar{\pi}(a)=\frac{a}{e} e^{-\frac{[1+a]}{a} \ln [1+a]} \\
\bar{W}(a)=\frac{a}{e}
\end{array} .\right.
$$

These two functions are strictly increasing over $\left[a_{\min }, a_{\max }\right]^{24}$. Hence,

$$
\left\{\begin{array}{c}
a^{m}=a^{s}=a_{\max } \\
p^{s}=e^{a_{\max }}<\left[1+a_{\max }\right] e^{a_{\max }}=p^{m}
\end{array} .\right.
$$

\footnotetext{
${ }^{24}$ One can check that $\bar{\pi}^{\prime}(a)=\frac{1}{a} e^{-1} \frac{\ln (a+1)}{(a+1)^{\frac{1}{a}(a+1)}}>0$ and $\bar{W}^{\prime}(a)=\frac{1}{e}>0$ for all $a \in\left[a_{\min }, a_{\max }\right]$
} 


\section{References}

[1] Amir, R. (1996). Sensitivity analysis in multisector optimal economic dynamics, Journal of Mathematical Economics, 25, 123-141.

[2] Amir, R., and Bloch, F. (2009). Comparative statics in a simple class of strategic market games, Games and Economic Behavior, 65, 7-24.

[3] Amir R. and Lambson, V. E. (2000). On the effects of entry in Cournot markets, Review of Economic Studies, 67, 235-254.

[4] André, F. J., González, P. and Porteiro, N. (2009). Strategic quality competition and the Porter Hypothesis, Journal of Environmental Economics and Management 57, 182-194.

[5] David, M. and B. Sinclair-Desgagné (2010). Pollution abatement subsidies and the eco-industry, Environmental and Resource Economics, 45, 271-282.

[6] Ebert, U. and von dem Hagen, O. (1998). Pigouvian taxes under imperfect competition If consumption depends on emissions, Environmental and Resource Economics, 12, 507-513.

[7] Gabszewicz, J. and J.-F. Thisse (1979), Price competition, quality, and income disparities, Journal of Economic Theory, 20, 340-359.

[8] Garcia-Gallego, A. and N. Georgantzis, 2009, Market effects of changes in consumers' social responsibility, Journal of Economics and Management Strategy, 18: 235-262.

[9] Johnson, J. P. and Myatt, D. P. (2016). On the simple economics of advertising, marketing, and product design, The American Economic Review, 96(3), 756-784.

[10] Kim, I. (2003). On the theory of the price- and quality-setting firm with uncertain demand. The Manchester School, 71, 626-640.

[11] Lambertini, L. and Tampieri, A. (2012a). Vertical differentiation in a Cournot industry : The Porter hypothesis and beyond, Resource and Energy Economics 34, 374-380.

[12] Lambertini, L. and Tampieri A. (2012b), Do minimum quality standards bite in polluting industries, Research in Economics 66(2), 184-194.

[13] Lombardini-Riipinen, C. (2005), Optimal tax policy under environmental quality competition, Environmental \& Resource Economics, 32, 317-336. 
[14] Moraga-Gonzalez, J. L. and N. Padron-Fumero (2002), Environmental policy in a green market, Environmental \& Resource Economics, 22, 419-447.

[15] Mantovani, A. and C. Vergari (2015). Hedonic vs environmental quality: which policy can help in lowering pollution emissions?, Mimeo, University of Bologna, Italy.

[16] OECD (2002). Report of the OECD workshop on information and consumer decisionmaking for sustainable consumption, Working Party on National Environmental Policy, ENV/EPOC/WPNEP(2001)16/FINAL, Paris.

[17] Sartzetakis, E. S., Xepapadeas A. and Petrakis, 2012, The role of information provision as a policy instrument to supplement environmental taxes, Environmental $\mathcal{E}$ Resource Economics, 52: $347-368$.

[18] Schmalensee, R. (1979), Market structure, durability, and quality: a selective survey, Economic Inquiry, 17, 177-196.

[19] Shaked, A. and J. Sutton (1982), Relaxing price competition through product differentiation, Review of Economic Studies, 49, 3-13.

[20] Sheshinski, E. (1976). Price, quality and quantity regulation in monopoly situations. Economica, 43, 127-137.

[21] Spence, A. M. (1975). Monopoly, quality, and regulation. The Bell Journal of Economics, 6, 417-429.

[22] Sutton, J. (1991). Sunk costs and market structure, The MIT Press, Cambridge, MA.

[23] Sutton, J. (1998). Technology and market structure, The MIT Press, Cambridge, MA.

[24] Varian, H. (1992). Microeconomic Analysis, Norton and Company, 3rd edition.

[25] Vives, X. (1999). Oligopoly pricing: Old ideas and new tools, The MIT Press. 